\title{
Congenital Bilateral C2 Transverse Foramina Stenosis Causing Adult-Onset Vertebrobasilar Insufficiency and Posterior Circulation Stroke
}

\author{
Ajeet Gordhan Catherine Lockhart \\ Department of Neuro-Endovascular Radiology, Advocate BroMenn Medical Center, \\ St. Joseph Medical Center, Bloomington, IN, USA
}

\section{Keywords}

Congenital bilateral C2 transverse foramina stenosis · Adult-onset vertebrobasilar insufficiency · Posterior circulation stroke · Computed tomography angiography · Episodic dizziness · Bow Hunter syndrome · Antiplatelet therapy

\begin{abstract}
Vertebrobasilar insufficiency leading to posterior circulation infarcts caused by congenital hypoplasia of the bilateral transverse foramina at the C2 level, affecting the caliber and flow of the bilateral distal cervical vertebral arteries in an adult, has not been previously reported. A 41-year-old male presented with episodic dizziness for a period of 1 year prior to consultation. Computed tomography angiography of the head and neck demonstrated congenital hypoplasia of the bilateral C2 transverse foramina, with absence of the vertebral arteries in each of the foramina and collateral reconstitution of diminutive intracranial vertebral artery segments. Brain MRI showed postinfarction encephalomalacia in the bilateral cerebellar hemispheres. The patient was considered not a surgical or endovascular candidate and was managed conservatively with antiplatelet therapy. Congenital anomalies of the bilateral cervical transverse foramina may present with vertebrobasilar insufficiency and infarction in adulthood.




\section{Case Reports in Neurology}

\section{Introduction}

Vertebrobasilar insufficiency and posterior circulation ischemic strokes can be difficult to diagnose and are more complex than carotid stenosis and anterior circulation strokes. Vertebrobasilar insufficiency typically presents with more vague complaints that cannot be easily distinguished from inner ear or cardiac diseases [1]. We report a case of protracted vertebrobasilar insufficiency and posterior circulation stroke caused by congenital bilateral C2 transverse foraminal stenosis.

\section{Case Report}

A 41-year-old male with a past medical history of coronary artery disease and multiple stent placement, obstructive sleep apnea, mixed hyperlipidemia, reactive airway disease, status post laparoscopic sleeve gastrectomy, depression, and seasonal allergic rhinitis presented with episodic dizziness. His symptoms began approximately 1 year prior to consultation. The first event was characterized by a slight feeling of vertigo, slowness of thinking, and an unsteadiness of gait. These symptoms lasted for approximately 2-3 days before resolution. He had an additional 3 episodes within the past year with similar symptoms that were not functionally limiting.

A computed tomography angiography (CTA) of the head and neck identified congenital bilateral C2 transverse foraminal stenosis with intraforaminal absence of the cervical vertebral arteries (Fig. 1a-c) and reconstitution of diminutive intracranial vertebral arteries from collaterals (Fig. 2a, b). A brain MRI showed areas of encephalomalacia in the inferior cerebellar hemispheres bilaterally compatible with small remote infarcts (Fig. 3a, b). The patient was treated with medical management that included Aspirin $325 \mathrm{mg}$ daily.

\section{Discussion}

Vertebrobasilar insufficiency typically presents with 1 or more of the following symptoms: vertigo or dizziness, syncope or near-syncope, motor or sensory deficits, dysarthria, ataxia, nystagmus, nausea with or without vomiting, Horner syndrome, visual blurring, diplopia, headache, and posterior circulation infarction [1-3]. The causes of vertebrobasilar insufficiency are multiple and range from intravascular etiologies, such as atherosclerosis and fibromuscular dysplasia, to extravascular etiologies, such as osteophytes, fascial bands, cervical musculature hypertrophy, and rarely osseous congenital anomalies of the cervical spine and vertebral artery [2].

Vertebrobasilar insufficiency may occur at any age but typically presents in the 5th to 7th decade. It affects males more often than females in a ratio of 2:1 and has a mean diagnosis age of $57 \pm 11$ years [4]. The etiology of extravascular compression of the vertebral artery is dependent on age [2]. Pediatric patients typically present with congenital anomalies, such as bony malformation at the atlantoaxial level or a suboccipital bony protuberance compressing the vertebral artery [2]. Juvenile patients typically present with occipital condylar bone spurs, anomalies of the cervical spine, or anomalous bypass of the vertebral artery. Adults typically present with abnormal bony formation, such as osteophytes, spondylotic changes, and idiopathic skeletal hyperostosis, or disc herniation, neck muscle hypertrophy, instability of the uncovertebral joint, and vertebral artery dissection [2]. Extravascular ver- 
tebral artery compression may also be acquired as a complication of cervical spine surgery [4].

In rotational occlusion of the vertebral artery (Bow Hunter syndrome), vertebrobasilar insufficiency is caused by proximal vertebral artery occlusion up to the C7 level (V1 segment) in $4 \%$ of the cases, by occlusion between C3 and C7 (V2 segment) in 58\% of cases, by occlusion between C1 and C2 (V3 segment) in 36\% of cases, and by occlusion distal to C1 (V4 segment) in $2 \%$ of cases [4]. The left vertebral artery is affected in $49.5 \%$, the right vertebral artery is affected in $36 \%$, and $13.7 \%$ of the cases are affected bilaterally. Of the cases with bilateral involvement, most are from abnormalities seen at different cervical levels and occur in the lower cervical spine, or they are from abnormalities that were acquired, such as osteophyte formation, and not from congenital anomalies [5-7]. Our case describes bilateral C2 congenital anomalies. The congenital variations of the cervical transverse foramina are characterized by alternate extraosseous pathways of the vertebral artery [8].

Vertebrobasilar insufficiency should be included in the differential diagnosis for patients with a complaint of episodic dizziness and vertigo, as it is often treatable and potentially reversible. Vascular imaging with CTA or magnetic resonance angiography is initially recommended [2]. Catheter-based angiography is the reference standard as it allows for better visualization of the cervical and cerebral vasculature and may be useful in identifying a postural component to vertebral artery flow limitation [1].

Conservative therapy for rotational vertebrobasilar insufficiency includes avoiding head and neck rotation and antiplatelet or anticoagulant therapy [2]. In 21 patients who received conservative management, 19 patients did not go on to have further posterior circulation strokes and 4 patients had spontaneous resolution of symptoms [2].

Surgical decompression of mass effect on the vertebral artery or cervical fusion or combination of decompression and fusion are the typical surgical modalities in the treatment of vertebrobasilar insufficiency from extravascular compression. In a study of 126 cases of rotational occlusion of the vertebral artery leading to vertebrobasilar insufficiency, $73 \%$ underwent decompressive surgery (V1 segment, 80\%; V2 segment, 83\%; V3/V4 segments, $60 \%$, while the remainder either had cervical fusion alone or less commonly decompression and fusion (V2 segment, 11\%; V3/V4 segment, 29\%). Of the patients that received decompression surgery, $87 \%$ were asymptomatic, and of those patients who received decompression and fusion, $100 \%$ were asymptomatic [4]. In bilateral compression of the vertebral arteries, a combination of decompression and fusion is recommended [2]. The surgical option for our patient was considered high risk due to potential injury to the paravertebral collateral vessels. The patient also refused surgical fusion of the $\mathrm{C} 1 / \mathrm{C} 2$ vertebrae due to mobility restriction concerns.

Endovascular treatment data are limited and are without clinical trials. There is a case series by Darkhabani et al. [9] of 4 patients that were treated with vertebral artery stenting, 3 in the dominant vertebral artery and 1 in the nondominant vertebral artery. In this study, all stents were placed in the C2-C6 segment. All 4 of the patients treated with this modality reported symptomatic relief. Given the degree and bilaterality of the bony stenosis, this modality was not considered a feasible option for our patient.

\section{Conclusion}

This case report demonstrates a rare cause of vertebrobasilar insufficiency and posterior circulation infarction. Bilateral congenital C2 transverse foraminal hypoplasia with seg- 


\section{Case Reports in Neurology}

mental vertebral artery agenesis resulting in adult-onset bilateral cerebellar infarction has not been previously described. A high clinical suspicion for vertebrobasilar insufficiency should be maintained for patients with intermittent positional dizziness (Bow Hunter syndrome).

\section{Statement of Ethics}

The authors have no ethical conflicts.

\section{Disclosure Statement}

The authors have no conflicts of interest to disclose.

\section{References}

1 Namini A, Naylor M, Koenigsberg RA: Vertebrobasilar insufficiency and stroke - a review of posterior circulation diagnostic imaging and endovascular treatment options. J Am Osteopath Coll Radiol 2015;4:15-23.

2 Duan G, Xu J, Shi J, Cao Y: Advances in the pathogenesis, diagnosis and treatment of Bow Hunter's syndrome: a comprehensive review of the literature. Interv Neurol 2016;5:29-38.

3 Park J, Lee C, You N, Kim S, Cho K: Cervicogenic vertigo treated by C1 transverse foramen decompression: a case report. Korean J Spine 2014;11:209-211.

4 Jost GF, Dailey AT: Bow Hunter's syndrome revisited: 2 new cases and literature review of 124 cases. Neurosurg Focus 2015;38:E7.

-5 Tsutsumi S, Ito M, Yasumoto Y: Simultaneous bilateral vertebral artery occlusion of the lower cervical spine manifesting as Bow Hunter's syndrome. Neurol Med Chir (Tokyo) 2008;48:90-94.

-6 Shinohara N, Kohno K, Takeda S, Ohta S, Sakaki S: A case of Bow Hunter's stroke caused by bilateral vertebral artery occlusive change on head rotation to the right. No Shinkei Geka 1998;26:417-422.

7 Fleming JB, Vora TK, Harrigan MR: Rare case of bilateral vertebral artery stenosis caused by C4-5 spondylotic changes manifesting with bilateral Bow Hunter's syndrome. World Neurosurg 2013;79:799.E1-E5.

-8 Zibis AH, Mitrousias V, Baxevanidou K, Hantes M, Karachalios T, Arvanitis D: Anatomical variations of the foramen transversarium in cervical vertebrae: findings, review of literature, and clinical significance during cervical spine surgery. Eur Spine J 2016;25:4132-4139.

-9 Darkhabani MZ, Thompson MC, Lazzaro MA, Taqi MA, Zaidat 00: Vertebral artery stenting for the treatment of Bow Hunter's syndrome: report of 4 cases. J Stroke Cerebrovasc Dis 2012;21:908.e1-e5. 


\section{Case Reports in Neurology}

\begin{tabular}{l|l}
\hline Case Rep Neurol 2017;9:137-142 \\
\hline DOI: $10.1159 / 000476031$ & $\begin{array}{l}\text { (c) 2017 The Author(s). Published by S. Karger AG, Basel } \\
\text { www.karger.com/crn }\end{array}$ \\
\hline
\end{tabular}

Gordhan and Lockhart: Congenital Bilateral C2 Transverse Foramina Stenosis Causing Adult-Onset Vertebrobasilar Insufficiency and Posterior Circulation Stroke
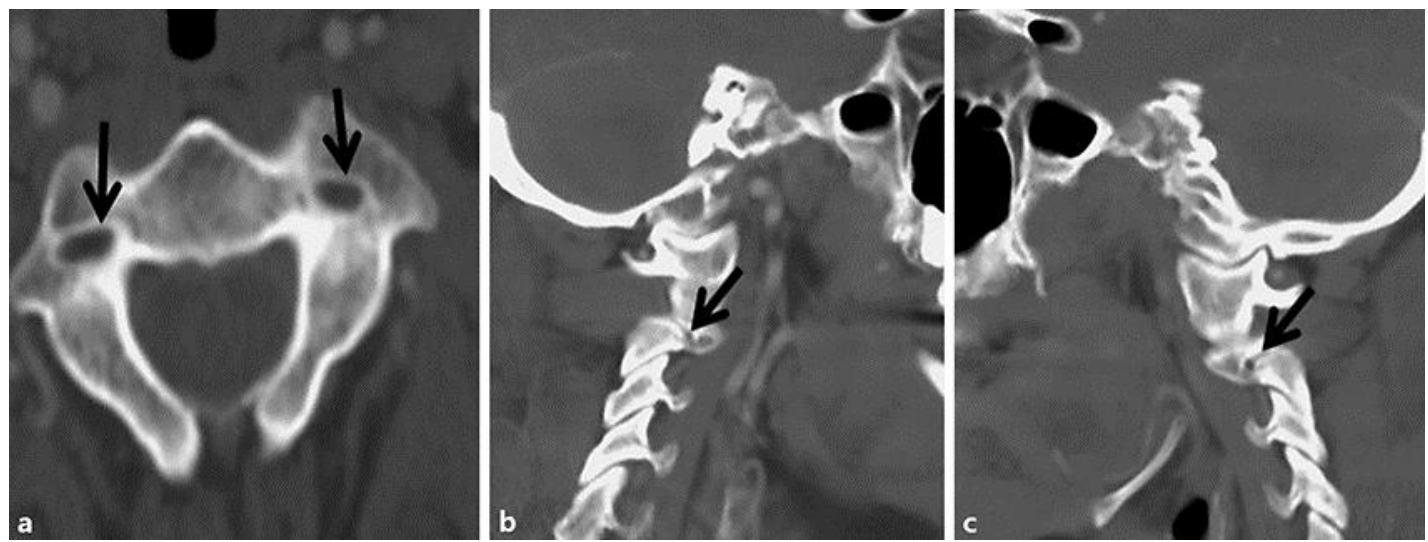

Fig. 1. Select axial (a) and paramedian sagittal right (b) and left (c) images of the neck computed tomography angiogram demonstrating bilateral hypoplastic transverse foramina at $\mathrm{C} 2$ with nonvisualization of the intraforaminal cervical vertebral artery segments (black arrows).
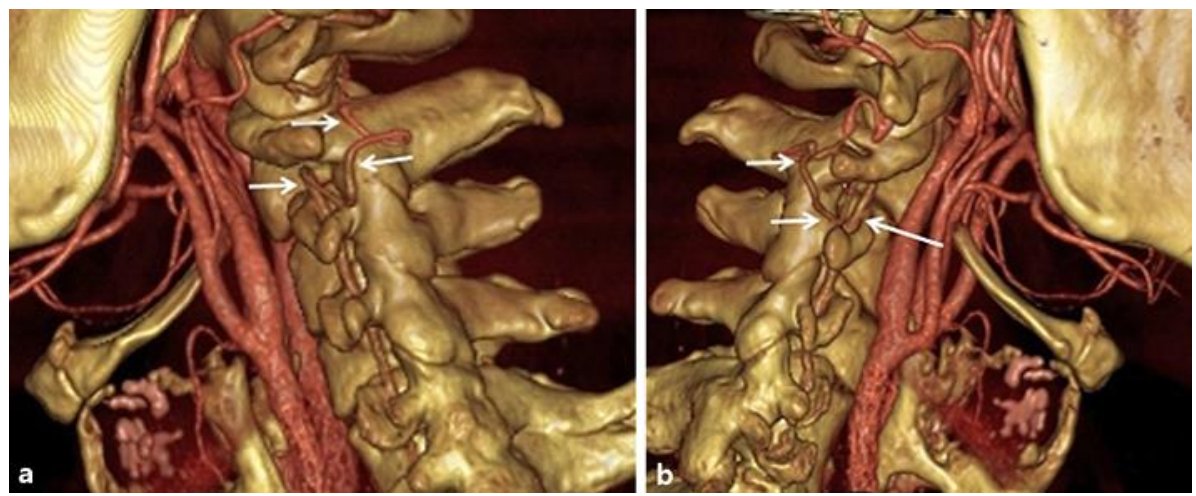

Fig. 2. Select 3D reformatted right lateral (a) and left lateral (b) images of the neck computed tomography angiogram demonstrating the course of the extraforaminal collateralized cervical vertebral artery at $\mathrm{C} 2$ level (white arrows). 


\section{Case Reports in Neurology}

Gordhan and Lockhart: Congenital Bilateral C2 Transverse Foramina Stenosis Causing Adult-Onset Vertebrobasilar Insufficiency and Posterior Circulation Stroke
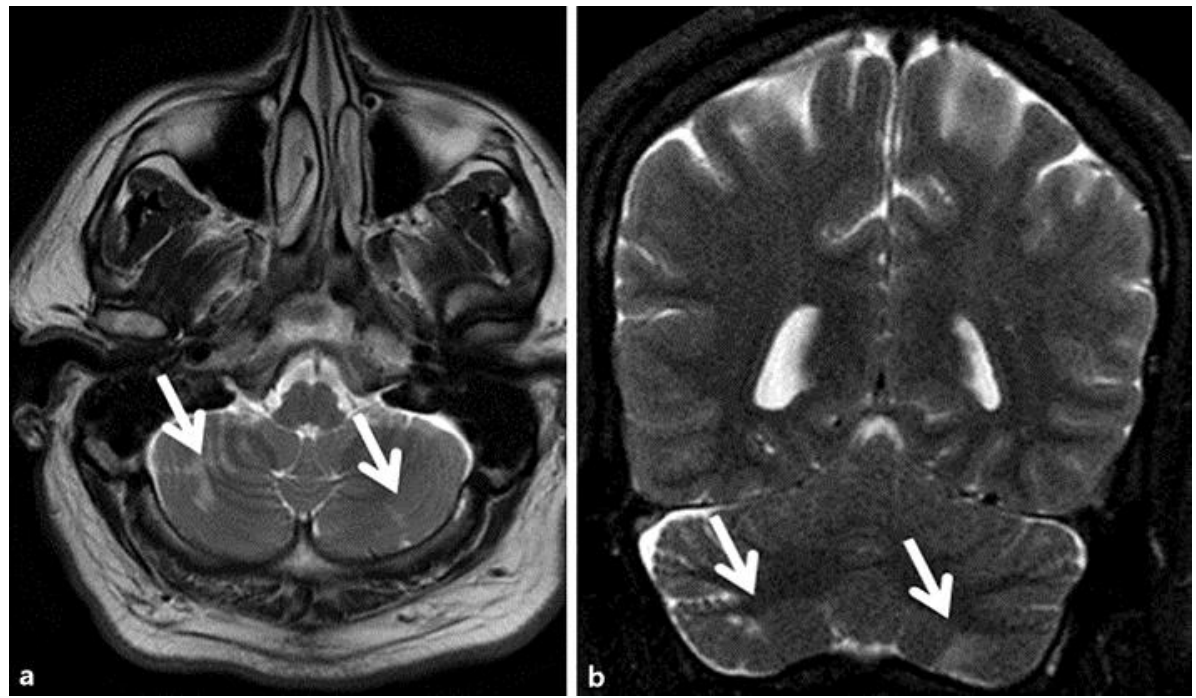

Fig. 3. Axial T2 (a) and coronal T2 (b) images of the brain MRI demonstrating established cortical and subcortical infarcts of the bilateral inferior cerebellar hemisphere (white arrows). 